\title{
Origin of enhanced gamma radiation in thunderclouds
}

\author{
A. Chilingarian $\odot,{ }^{1,2,3}$ G. Hovsepyan, ${ }^{1}$ A. Elbekian, ${ }^{1}$ T. Karapetyan, ${ }^{1}$ L. Kozliner, ${ }^{1}$ H. Martoian, ${ }^{1}$ and B. Sargsyan ${ }^{1}$ \\ ${ }^{1}$ A. Alikhanyan National Laboratory (Yerevan Physics Institute), Yerevan 0036, Armenia \\ ${ }^{2}$ National Research Nuclear University MEPhI, Moscow 115409, Russia \\ ${ }^{3}$ Space Research Institute of RAS, Moscow 117997, Russia
}

(Received 17 September 2019; published 11 December 2019)

\begin{abstract}
Natural gamma radiation (NGR), one of the major geophysical parameters directly connected with cloud electrification and lightning initiation, is highly enhanced during thunderstorms. At low energies below $3 \mathrm{MeV}$, the enhancement of NGR is due to natural isotope radiation, and for energies up to $50 \mathrm{MeV}$, it is due to the operation of the newly discovered electron accelerators in the thunderclouds. For the first time, we present a comprehensive model of the enhanced fluxes of radiation incident on the earth's surface during thunderstorms. In addition to the already explained minute-long fluxes of high-energy electrons and gamma rays from relativistic runaway electron avalanches (RREA), we clarify also the origin of hour-long isotropic fluxes of low-energy gamma rays from the Rn-222 progenies. Also, as a direct evidence of RREA, we present photographs of optical emission during the development of electron-gamma ray cascades in the atmosphere. Natural radioactivity is a source of continuous exposure of human beings to radiation. Radiation protection of living organisms requires an understanding of all sources and possible ways of enhancement of the radiation levels that can double for several hours in the energy domain of hundreds of $\mathrm{keV}$. Therefore individual irradiation doses can be exceeded during thunderstorms. The models used for the forecasting of thunderstorms and other severe atmospheric phenomena need an accurate account of the ionizing radiation in the atmosphere. The airglows can influence the operation of optical, fluorescence, and atmospheric Cherenkov telescopes and fluorescence detectors.
\end{abstract}

DOI: 10.1103/PhysRevResearch.1.033167

\section{INTRODUCTION}

In Refs. [1,2], we describe a new phenomenon in highenergy atmospheric physics, namely, a flux of electrons and gamma rays lasting for several hours that correlates with a thunderstorm and smoothly decays after cessation of a storm. Such enhancement of the particle flux in the thunderous atmosphere was well documented during the last two decades. Seed electrons from an ambient population of cosmic rays are accelerated in the strong electric field forming an electrongamma ray avalanche, directed either downwards to the Earth's surface or upwards into the open space, depending on the direction of the electric field. Intense fluxes of gamma rays observed in space are called terrestrial gamma flashes (TGFs) [3-5]; the ones in the atmosphere are called gamma glows [6-9], and the ones that are observed on the ground are called thunderstorm ground enhancements (TGEs) [10-16]. In the latter, also neutron fluxes are observed [17-20]. The runaway breakdown (RB) [21], also referred to as relativistic runaway electron avalanche (RREA) [22-24], and the modification of electron energy spectra (MOS) [25] are the only theoretical models satisfactorily explaining electron acceleration up to

Published by the American Physical Society under the terms of the Creative Commons Attribution 4.0 International license. Further distribution of this work must maintain attribution to the author(s) and the published article's title, journal citation, and DOI.
40-50 MeV (see Fig. 1). The MOS process, which occurs when the strength of the atmospheric electric field is below the breakeven field value at which avalanches are developed, can enhance the gamma ray flux only by few percent. A systematic investigation of TGE phenomena at Aragats space environment center (ASEC) was performed with plastic scintillators previously used for the research of galactic cosmic rays and solar and space weather phenomena. The energy threshold of these detectors exceeds $5 \mathrm{MeV}$ and episodes of enhanced flux do not surpass 10 minutes [26]. However, after the installation of NaI spectrometers [27] with a lower energy threshold on the Aragats network, during continuous monitoring of particle fluxes [28], we observe a natural gamma radiation in hundreds of keV energy range as well. By measuring the differential energy spectra of electrons and gamma rays in the energy range from $300 \mathrm{keV}$ to $50 \mathrm{MeV}$, we discovered that the particle fluxes continued for $\sim 4$ hours with a characteristic decay time of $\sim 1$ hour. As the half-life time of the flux decay well coincides with the half-life time of isotopes ${ }^{214} \mathrm{~Pb}(\sim 300 \mathrm{keV})$ and ${ }^{214} \mathrm{Bi}(\sim 600 \mathrm{keV})$ of the uranium-radium decay chain, first of all, we check the hypothesis of the precipitation origin of enhanced gamma ray flux. By observing numerous TGEs without any precipitation, we reject this hypothesis [29]. We also reject the hypothesis of the TGE origin from the radon progenies gathered in the thunderclouds suggested in Ref. [30] by observing TGEs from clouds with rather a high base $(>500 \mathrm{~m})$, and by detecting gamma rays with energies well above the isotope decay energies $[31,32]$. Nonetheless, an explanation of the long-lasting TGEs was still missing. 


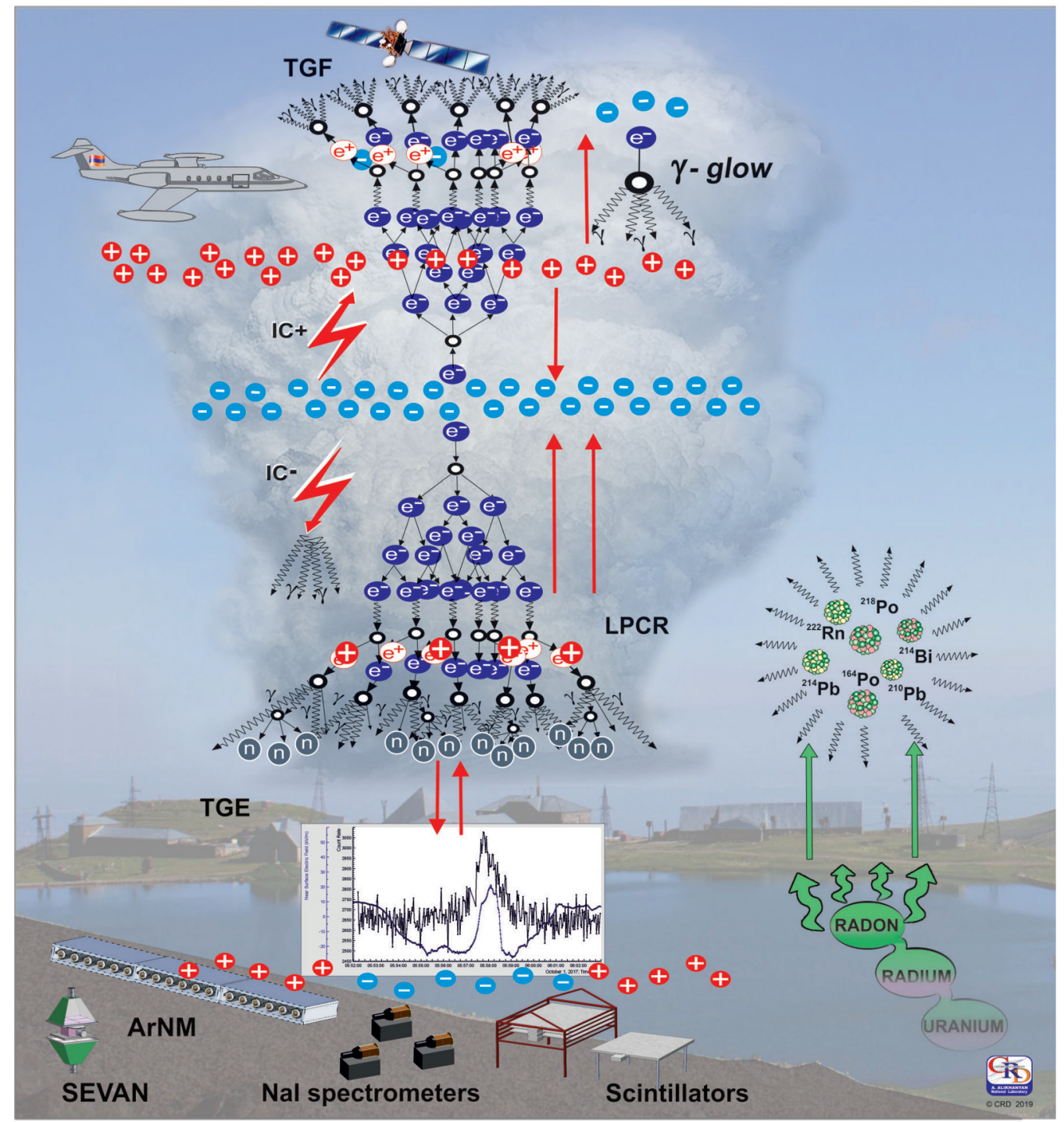

FIG. 1. A schematic view of the NGR enhancement during thunderstorms.

In the early work [33], it was mentioned that "radondaughter ions are found to disappear almost completely at ground level under an active thunderstorm due to upward migration of the ions under the influence of strong electric fields." In Ref. [34], a strong correlation between gamma ray levels, precipitation, and the vertical component of the nearsurface electric field was measured. In many other studies, it was observed that radon and its progenies are very mobile and readily attach to aerosol surfaces. Thus emanated radon progenies become airborne and immediately attach to the dust particles and aerosols existing in the atmosphere and are lifted by the near-surface electric field upward providing isotropy radiation of low-energy gamma rays (see the right side of Fig. 1). Owing to their long half-life (27 and 20 minutes), ${ }^{214} \mathrm{~Pb}$ and ${ }^{214} \mathrm{Bi}$ are the most abundant radon progenies in the atmosphere and candidates for the NGR at low energies.

In the summer of 2019, we performed several experiments with $\mathrm{NaI}$ spectrometers to reveal the contribution of Rn progenies to observed TGEs. The measured energy and inclination of the enhanced low-energy particle fluxes validate the idea that the origin of these fluxes is gamma radiation of $\mathrm{Rn}$ progenies lifted to the atmosphere by the near-surface electric field. Also, we present the first optical signature of RREA developed in the lower part of the thundercloud.

\section{ELECTRON ACCELERATOR OPERATING IN THE ATMOSPHERE}

As we can see in Fig. 1, the electron acceleration towards Earth is due to the electric field between the main negative (MN) charge region in the middle of the cloud and the positive charge induced on the ground. Inside the thundercloud, this field can be significantly increased by an emerging lower positively charged layer (LPCR) located below the main negative charge region.

The electric field in the gap between the MN layer and LPCR will be enhanced by the superposition of $E_{\mathrm{LPCR}}$ and $E_{\mathrm{MN}}$, while the field below the LPCR will be reduced due to the opposite directions of $E_{\mathrm{LPCR}}$ and $E_{\mathrm{MN}}$. The maximal intensity (and maximal energy of TGE particles) is observed when 


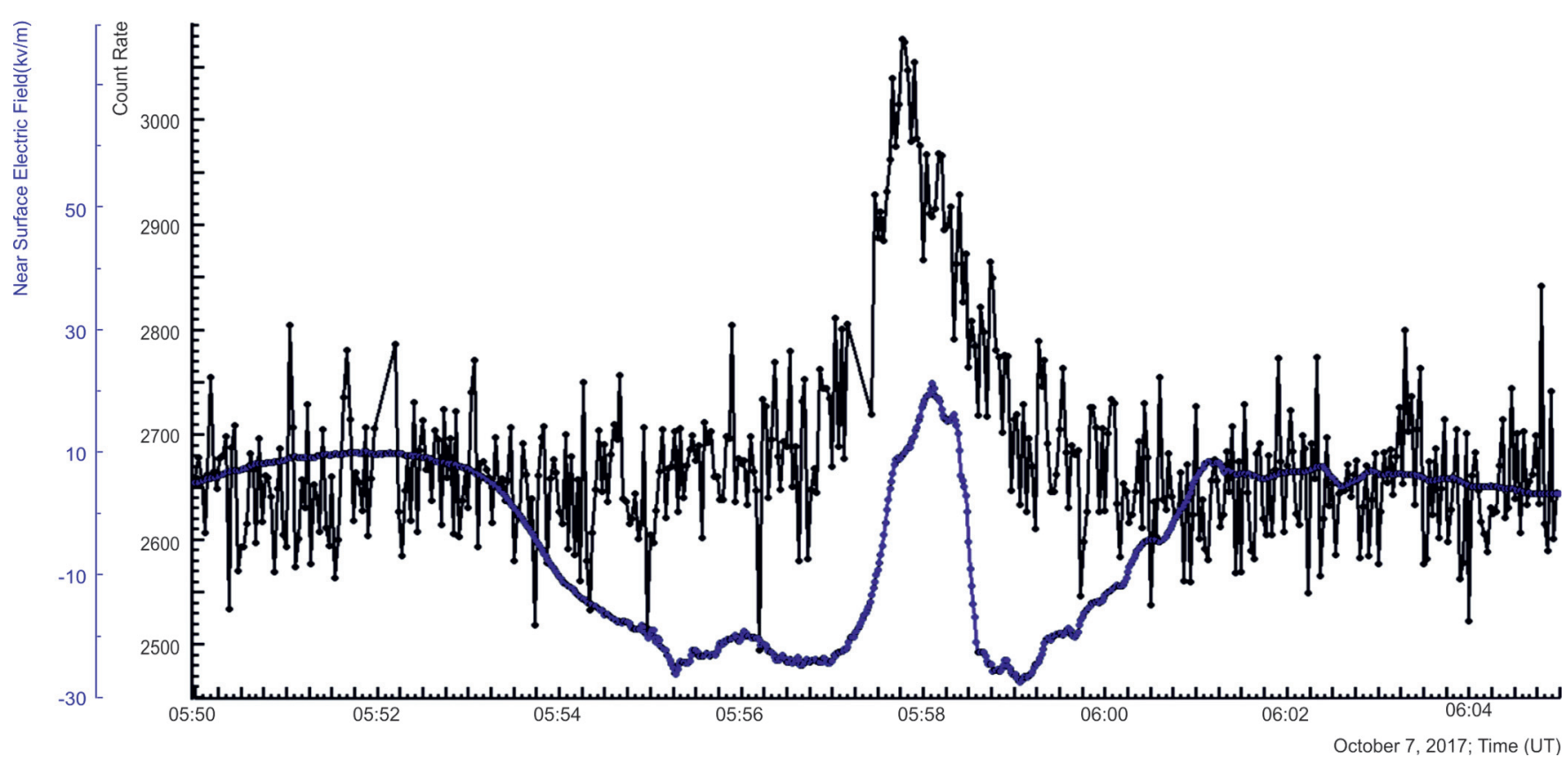

FIG. 2. The disturbances of near-surface electric field and corresponding count rate enhancement registered by the 60-cm-thick plastic scintillator. The maximum particle flux coincides with a positive "bump" in the electrostatic field time series.

the strength of the local electric field in the cloud exceeds the breakeven threshold and RB/RRE avalanches start to develop downward. For the operation of the electron accelerator and detection of TGE we need (1) a proper (breakeven) electric field $\left(E_{\mathrm{LPCR}}+E_{\mathrm{MN}}\right)$, for instance, $E>1.7 \mathrm{kV} / \mathrm{m}$, in the gap located somewhere between 3500-5000 m above Earth's surface, and (2) a large spatial extent of the field (larger than 500 $\mathrm{m})$.

From the observed patterns of electrostatic field disturbances during the TGE occurrences, we assume that rising "bumps" in the time series (see Fig. 2, the zoomed version of the schematic chart shown in the Fig. 1 below the thundercloud) are an essential characteristic of the thunderstorm, evidencing vertical movement of positive hydrometeors in the direction towards the Earth's surface and causing excursion of the polarity of the near-surface electric field. Consequently, enlarging the spatial extent of the field leads to a larger potential drop and acceleration of electrons to high energies, up to $50 \mathrm{MeV}$. The maximum of experimentally measured particle flux (top black curve in Fig. 2) coincides with the "bump" rising from the deep negative electrostatic field and reaching the positive domain (bottom blue curve in Fig. 2). However, the large variability in duration, amplitude, and shape of TGEs detected by ASEC facilities, as well as, fluctuating patterns of the near-surface electrostatic field disturbances, allows also for other scenarios of the emergence of an electric field strong enough to accelerate electrons downwards. Recently, it was discovered that downward RREA (named gamma glow) occurred also between the negative screening layer and the upper positive charge layer just below it $[8,35]$. It is interesting to mention as well that examining the general characteristics of the preliminary breakdown and stepped leader processes, the authors of Ref. [36] notice that the combination of a wide middle negative charge region and a small lower positive charge region is a favorable condition for the origination of intense return strokes. The same conditions support the occurrence of intense TGE that usually was followed by the lightning flash [37].

\section{ENHANCEMENT OF THE NATURAL GAMMA RADIATION DURING THUNDERSTORMS}

In addition to the well-proven "electrical" origin of the high-energy atmospheric phenomena that can enhance particle flux approximately ten times above the background level (see Fig. 18 of Ref. [29]), in Refs. [31,32], we confirm that "radon progenies radiation significantly contributes to the count rate enhancements in the energy range below $3 \mathrm{MeV}$." However, the mechanism of this phenomenon remains unknown.

To reveal this enigmatic contribution of Rn progenies to TGE flux, we monitor the NGR with a network of NaI spectrometers. NaI crystals of size $12 \times 12 \times 24 \mathrm{~cm}$ (relative energy resolution, FWHL is $\sim 50 \%$ ) register not only time series of count rates, but also time series of the 1-minute histograms of energy releases. The low energy threshold $(\sim 300 \mathrm{keV})$ provides large statistics $(\sim 50000$ counts in a minute) for recovering differential energy spectra (see details of spectrometer operation in Ref. [27]). To investigate the origin of low energy gamma ray fluxes, we cover some spectrometers with lead filters. First of all, we put spectrometers on the lead to prove that the TGE flux comes from the top and sides of the crystal, and not from the bottom. Then, covering spectrometers from the top, we prove that the low energy portion of TGE comes under large zenith angles. The high-energy portion of TGE comes only from the near-vertical direction due to the vertical alignment of the atmospheric electric field.

After rather dry spring-summer seasons on Aragats, the September thunderstorms are highly intensified in the first 


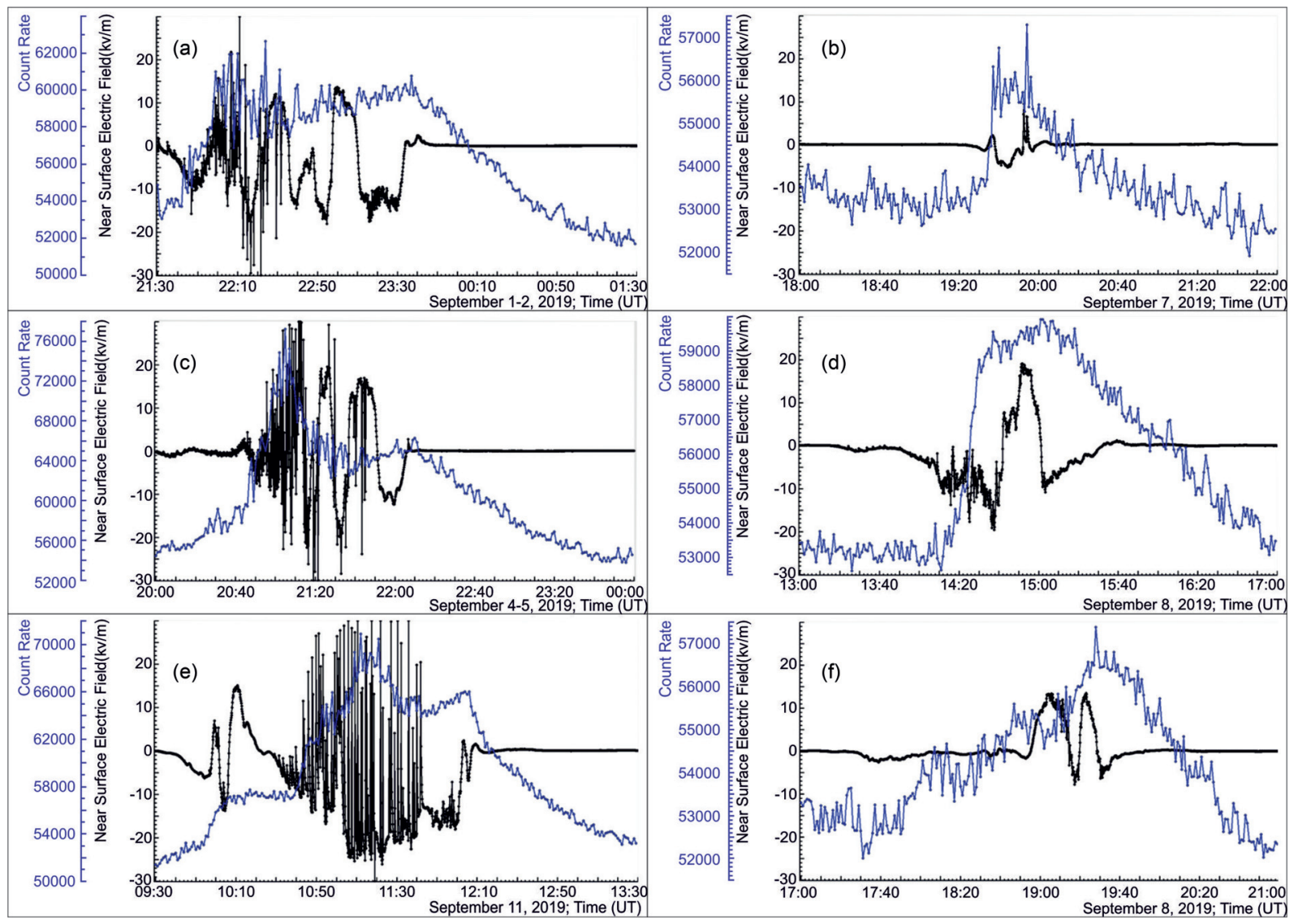

FIG. 3. One-minute time series of the disturbances of the near-surface electric field (black) and the particle flux enhancement and decay (blue) measured by a large NaI spectrometer in the first decade of September 2019. The duration of TGES shown in all frames is $\sim 4$ hours; the whole scale of the electric field is kept in $-30-+30 \mathrm{kV} / \mathrm{m}$ interval.

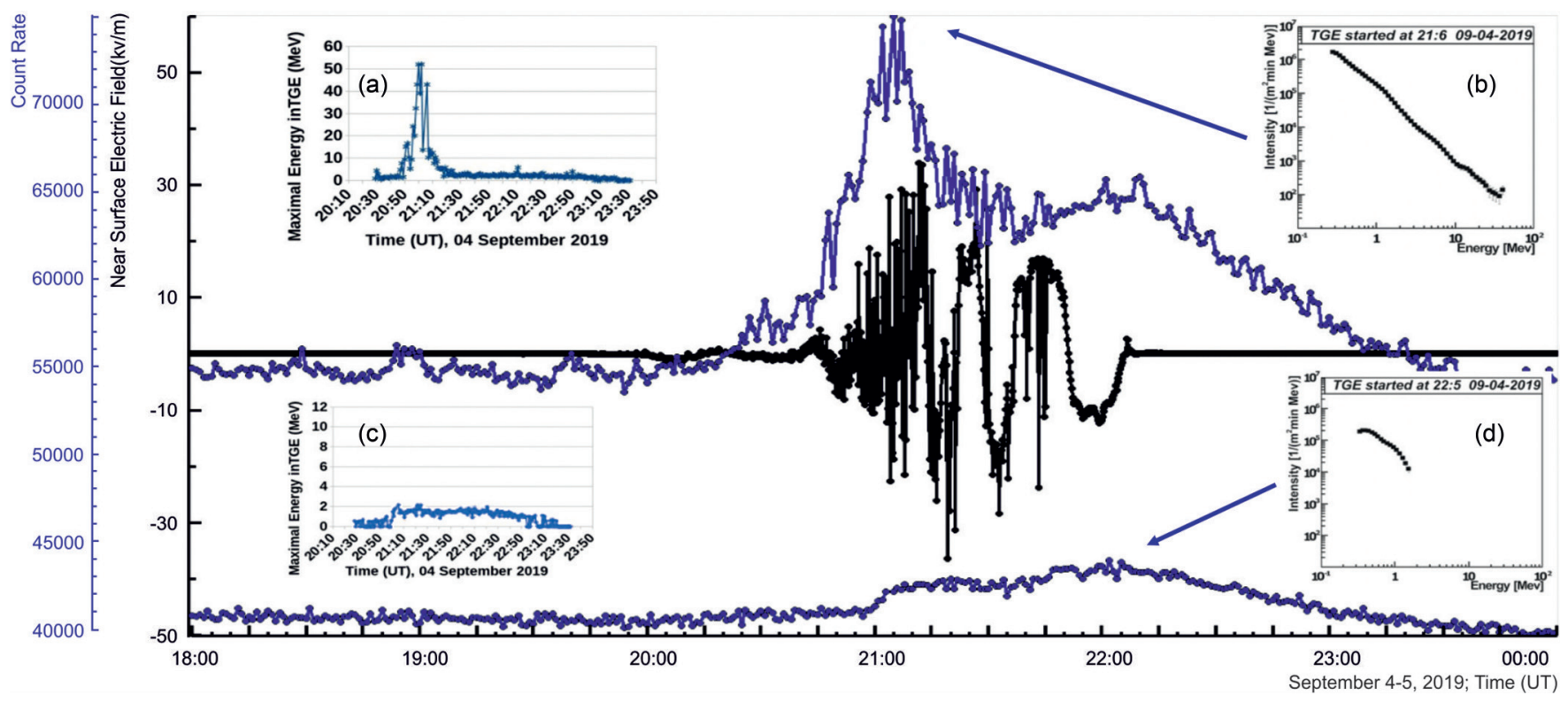

FIG. 4. One-minute time series of TGE measured on September 4 [see Fig. 2(c)] by NaI spectrometers with the lead filter on top (bottom blue curve) and without lead (top blue curve). The disturbances of the near-surface electric field are shown between these curves (black). In insets (a) and (c), we show the histogram of maximal energies of energy spectra measured each minute by both spectrometers, and in insets (b) and (d), examples of measured energy spectra. 

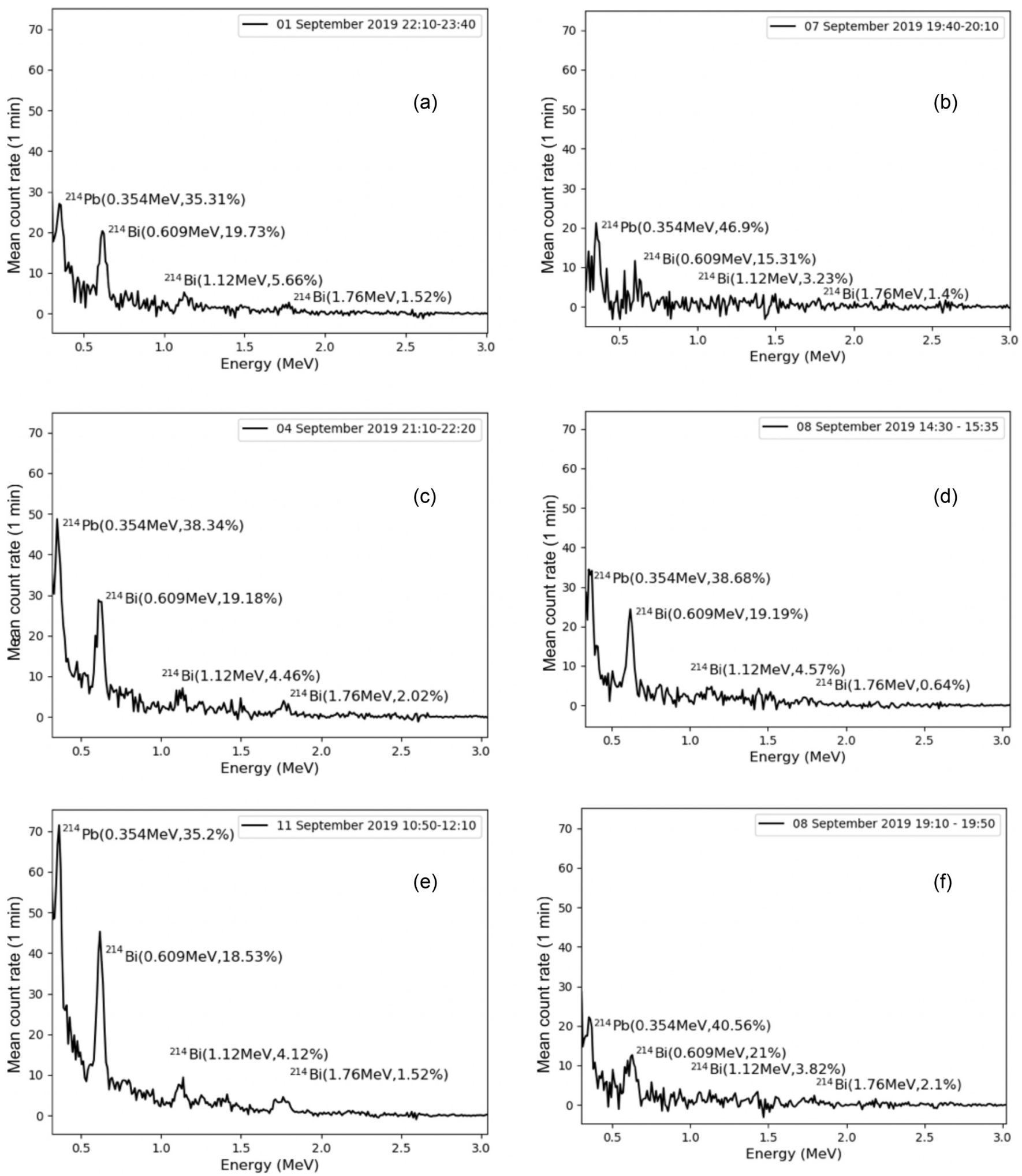

FIG. 5. Spectrograms of the Rn progenies, the gamma radiation of which is responsible for the enhancement of the count rate at low energies during thunderstorms. The same TGE events as shown in Fig. 2, but observed by a precise spectrometer with high resolution (FWHH $\sim 7.7 \%$ ).

decade producing plenty of TGEs, adding from $5 \%$ to $50 \%$ to the fair-weather count rate. All storms lasted $\sim 3$ hours, with a characteristic decay lasting $\sim 1$ hour afterward. The near-surface electric field disturbances, measured with an
EFM-100 electric mill varied from 4 to $25 \mathrm{kV} / \mathrm{m}$. In Fig. 3, we demonstrate six TGEs with a rather smooth decay of the count rate at the end of the storm. A spectrometer with a lead filter on the top measures only isotropic inclined flux from 

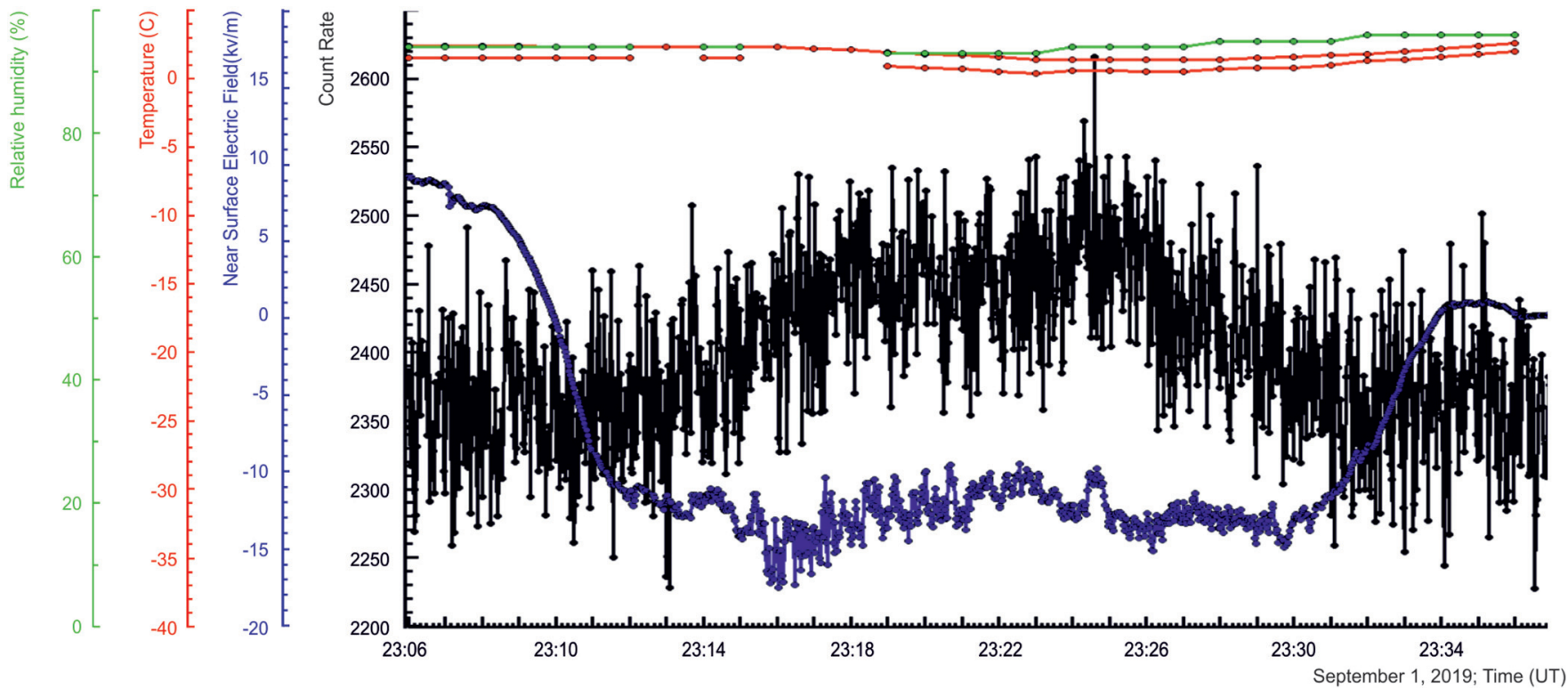

FIG. 6. The enhanced particle flux measured by the 5 -cm-thick, $4-\mathrm{m}^{2}$-area plastic scintillator (black curve). Energy threshold $\sim 5 \mathrm{MeV}$. Disturbances of the near-surface electric field measured by the electric mill EFM-100 (blue curve). By the outside temperature and dew point, we estimate the height of the cloud base approximately at $100 \mathrm{~m}$. Relative humidity was $93 \%$, no rain occurred.

$\mathrm{Rn}-222$ progenies gamma radiation. As the storm finishes, the electric field strength returns to fair-weather value, and the boosted uplift of $\mathrm{Rn}$ progenies stops. The half-life of count rate decay (20-35 minutes) fits well the half-life of the most-abundant gamma emitters from the Rn chain, namely, ${ }^{214} \mathrm{~Pb}$ (the half-live is 27 minutes) and ${ }^{214} \mathrm{Bi}$ (the half-live is 20 minutes). Sure, we cannot expect exact concurrence of TGE half-life and the isotope half-life: different isotopes appear in the atmosphere in a slightly different time, there are various decay modes with different branching ratios; the processes in the atmosphere are very dynamic, dependent on precipitation, wind, temperature, and electric field fast changes due to lightning flashes.

In Fig. 4, we show the time series of count rates measured by $\mathrm{NaI}$ spectrometers $\mathrm{N} 2$ (upper curve) and $\mathrm{N} 4$ (lower curve, $4 \mathrm{~cm}$ lead on the top). Between these curves, the disturbances of near-surface electric field measured by electric mill EFM-100 are shown. In the insets to the left [(a) and (c)], we demonstrate the time series of maximal energies of the recovered differential energy spectra for each minute of TGE. In the right insets [(b) and (d)], we demonstrate the examples of these one-minute energy spectra for both spectrometers.

The 50-MeV peak near 21:00 seen in insets 4(a) and 4(b) corresponds to high-energy gamma rays from RREA developed in the thunderous atmosphere above the detector. Both RREA and MOS processes produced a near-vertical flux of gamma rays. The maximal energies measured by the spectrometer with a lead on the top [isotropic gamma rays from radon progenies decay, see Figs. 4(c) and 4(d)] never exceeded $2 \mathrm{MeV}$.

In Fig. 5, we show the energy spectra of the same six TGEs shown in Fig. 3, now measured by a precise spectrometer ORTEC- 905-4 with 1024 channels, high stability, and much better (compared with the large NaI crystals) relative energy resolution (FWHM $\sim 7.7 \%$ at $0.3-2 \mathrm{MeV}$ energies). The four largest peaks in the spectrograms correspond to ${ }^{214} \mathrm{~Pb}$
(354 keV), ${ }^{214} \mathrm{Bi}(609 \mathrm{keV}),{ }^{214} \mathrm{Bi}(1120 \mathrm{keV})$, and ${ }^{214} \mathrm{Bi}$ $(1750 \mathrm{keV})$. The rest of the count rate can be attributed to other gamma radiating isotopes, a small portion of the lowenergy inclined gamma rays from the cosmic ray population, and scattered in the crystal gamma rays. Due to the small size of the NaI crystal $(5 \times 5 \times 5 \mathrm{~cm})$ of the ORTEC spectrometer, Compton scattered gamma rays form a continuous spectrum on the left of the isotope gamma radiation lines. Although the intensity of the main isotope peaks drastically changed due to variable meteorological conditions and different strength of near-surface electric field [compare Figs. 3(e) and 3(f), 5(e) and 5(f)], the abundances of isotopes are rather stable: $39.2 \%+/-4.4$ for ${ }^{214} \mathrm{~Pb}$ and $30.8 \%+/-3.4$ for ${ }^{214} \mathrm{Bi}$. Thus the overwhelming contribution of the ${ }^{214} \mathrm{~Pb}$ and ${ }^{214} \mathrm{Bi}$ isotopes confirms our scenario of the origin of the low energy NGR enhancement during thunderstorms.

\section{OPTICAL EMISSIONS PRODUCED BY RELATIVISTIC RUNAWAY ELECTRON AVALANCHES IN THE SKIES ABOVE ARAGATS}

Electrons generated during the development of RREA excite air molecules which then emit fluorescence light in the visible range. The number of emitted fluorescence photons is proportional to the energy deposited in the atmosphere by traversing electrons. Numerical models of fluorescence emissions for the electron-photon avalanches developing in the upper dipole of the thundercloud were described in Ref. [38]. The upward directed gamma rays originating in these avalanches produce terrestrial gamma ray flashes observed by the orbiting gamma ray observatories. Modeling results indicate that TGFs are most likely accompanied by detectable levels of optical emissions. Similarly, if the primary and secondary electrons can generate optical emissions by the radiative relaxation of excited atoms and molecules in the sparse upper atmosphere, the probability to detect airglows in 

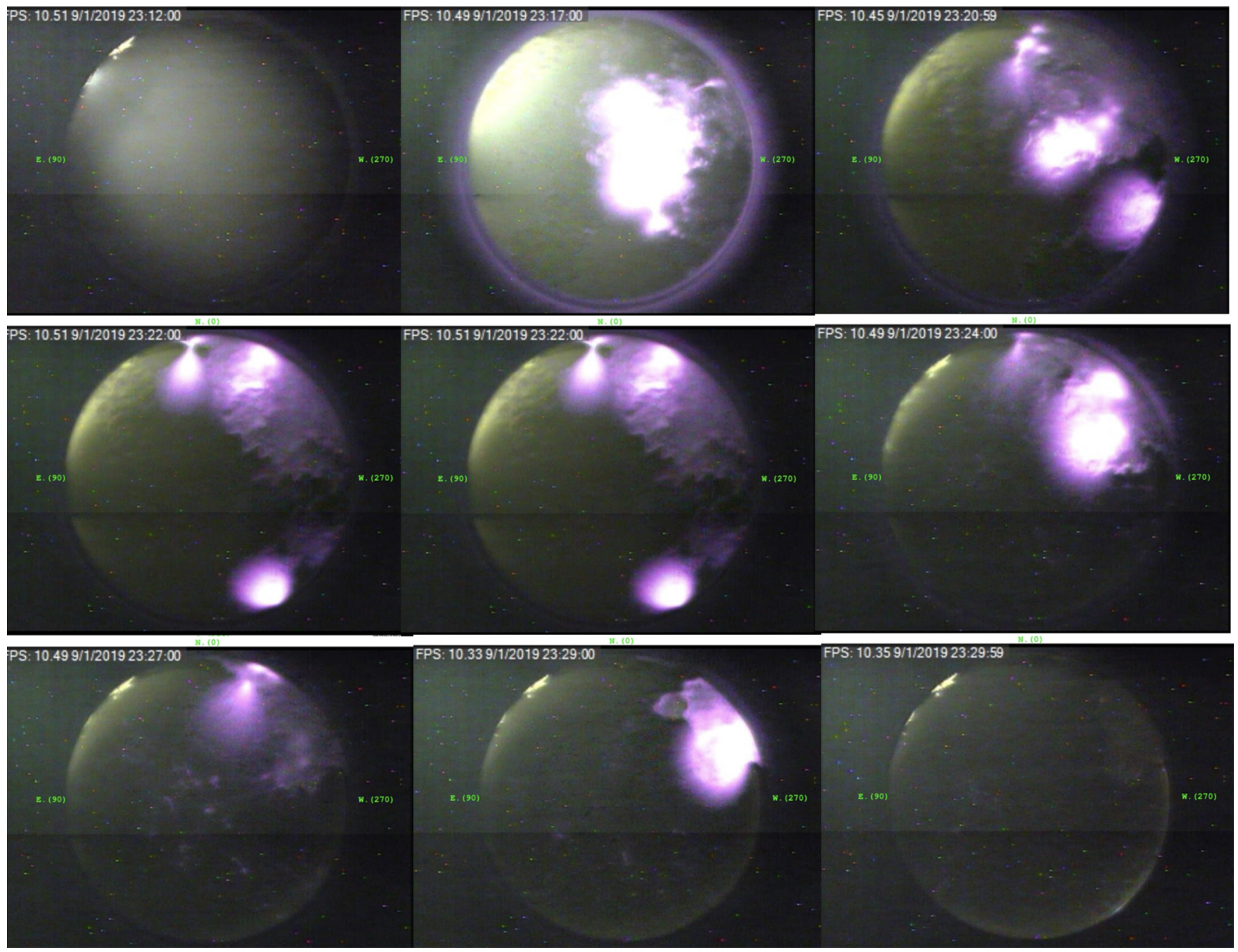

FIG. 7. Optical emission of the RREAs developing in the large-scale homogeneous electric field within the thundercloud. Randomly selected frames from the 15-minute-long continuous light bursts occurred on the 1st of September 2019. The clip covering the evolution of the airglow is available from the link [42].

the dense atmosphere where lower dipole is located is much higher.

Starting from 2014, we monitor the entire sky above Aragats with the "All Sky Cam" panoramic camera. The camera employs a color 1/3" Sony super HAD CCD II image sensor that has high sensitivity in the visible wavelength band of 300-700 nm [39].

Using a software package provided by the firm, we upload image streams to the internet and store digitized images in the ADEI database [40]. On strong storm conditions (when the near-surface electric field is enhanced by $5 \mathrm{kV} / \mathrm{m}$ ), we store images each second, elsewhere each minute.

In Ref. [41], we describe a 4-minute lasting airglow, which we relate to fluorescence emission from air molecules excited by high-energy electrons accelerated in the lower dipole of an electrified thundercloud. On the 1st of September 2019, we detected a 15-minute lasting uninterruptable airglow, now documented by $1-\mathrm{Hz}$ frequency shots of the same panoramic camera. Evidence of the "electron" origin of optical bursts is provided by the significant flux of TGE registered in the same 15 minutes (see Fig. 6).
In Fig. 7, we post some frames from long series of optical bursts detected by the panoramic camera located on the roof of the main building on Aragats research station, $3200 \mathrm{~m}$ above sea level. The detailed pattern of airglow digitized every 5 seconds can be seen in a clip by following the link [42]. Each glowing patch of optical emission in the frames corresponds to the relativistic runaway electron avalanche developed in the thunderous atmosphere above the station. As the cloud base is located $\sim 100 \mathrm{~m}$ above the earth's surface, the airglows are well seen in each frame. RREA glows should be much more intense as compared with extensive air shower (EAS) glows, light from which is registered by the fluorescence detectors of Pierre Auger experiment to estimate the energy of the ultrahigh cosmic rays [43].

\section{CONCLUSIONS}

Based on measurements of the intensity and energy spectra of NGR on Mt. Aragats, we present a comprehensive model explaining NGR enhancement during thunderstorms. We describe the TGE phenomenon as a mixture of two separate 
processes, both having roots in the electric fields (intracloud and near-surface) emerging during thunderstorms. For the first time, we observe the airglows initiated by electron-gamma ray avalanches developing in the lower atmosphere.

The temporal evolution of the long-lasting TGEs measured by particle detectors with a low threshold $(\sim 300 \mathrm{keV})$ is controlled by three processes: RREA, MOS, and Rn-220 progenies gamma radiation. The first two are connected with avalanche processes in the lower dipole (runaway electrons) and modification of the cosmic ray electron energy spectrum, the third, essential only at low energies, with natural radioactivity, is enormously activated during thunderstorms. This scenario of NGR origination is supported by measurements of the count rates and energy spectra of vertical and inclined gamma rays at Aragats. Thus our model is supported by the abundance of $\mathrm{Rn}$ progenies in the TGE energy spectrum at low energies, by the half-life of TGE, and by the absence of high energies in the measured flux of "inclined" low-energy gamma rays.

We present evidence that electron fluxes in the lower dipole are capable to generate well seen long-lasting optical emissions. It is interesting to mention that NASA's Cassini spacecraft has spotted a glowing patch of violet light near Saturn's north pole that marks the presence of an electrical circuit that connects Saturn with its moon Enceladus [44].
Now, the airglows from the electron beams are detected also on Earth.

Thus, first, we specify TGE phenomena by detecting simultaneous flux of high-energy electrons, gamma rays, and neutrons [11]; then, we observe RREA by detecting particle showers coming from clouds (extensive cloud showers [12]); next, we prove the existence of the lower dipole, which accelerates electrons downward [45]. In the same year, we performed simulations of the electron propagation in strong atmospheric electric fields, confirming the runaway phenomena [25]. Only in 2019, we presented a comprehensive model of TGE and direct optical evidence of RREA origination in the thunderous atmosphere.

\section{ACKNOWLEDGMENTS}

We thank the staff of the Aragats Space Environmental Center for the operation of the NaI network on Aragats. A. C. thanks M. Panasuk, A. Avetisyan, and R. Mirzoyan for useful discussions. The data for this study are available by the multivariate visualization software ADEI on the web page of the Cosmic Ray Division (CRD) of the Yerevan Physics Institute, http://adei.crd.yerphi.am/adei. We appreciate the support of the Russian Science Foundation Grant, Project No. 17-1201439.
[1] A. Chilingarian, G. Hovsepyan, S. Soghomonyan, M. Zazyan, and M. Zelenyy, Structures of the intracloud electric field supporting origin of long-lasting thunderstorm ground enhancements, Phys. Rev. D 98, 082001 (2018).

[2] A. Chilingarian, H. Mkrtchyan, G. Karapetyan, S. Chilingaryan, B. Sargsyan, and A. Arestakesyan, Catalog of 2017 Thunderstorm Ground Enhancement (TGE) events observed on Aragats, Sci. Rep. 9, 6253 (2019).

[3] G. Fishman, P. Bhat, V. Mallozzi, J. M. Horack, T. Koshut, C. Kouveliotou, G. N. Pendleton, C. A. Meegan, R. B. Wilson, W. S. Paciesas, S. J. Goodman, and H. J. Christian, Discovery of intense gamma ray flashes of atmospheric origin, Science 264, 1313 (1994).

[4] M. Briggs, V. Connaughton, C. Wilson-Hodge, R. D. Preece, G. J. Fishman, R. M. Kippen, P. N. Bhat, W. S. Paciesas, V. L. Chaplin, C. A. Meegan, A. von Kienlin, J. Greiner, J. R. Dwyer, and D. M. Smith, Electron-positron beams from terrestrial lightning observed with Fermi GBM, Geophys. Res. Lett. 38, L02808 (2011).

[5] M. Tavani et al. (AGILE Team), Terrestrial Gamma-Ray Flashes as Powerful Particle Accelerators, Phys. Rev. Lett. 106, 018501 (2011).

[6] M. McCarthy and G. Parks, Further observations of Xrays inside thunderstorms, Geophys. Res. Lett. 97, 5857 (1985).

[7] K. Eack, W. Beasley, W. Rust, T. C. Marshall, and M. Stolzenburg, Initial results from simultaneous observations of $\mathrm{X}$ rays and electric fields in a thunderstorm, J. Geophys. Res. 101, 29637 (1996).

[8] N. Kelley, D. Smith, J. Dwyer, M. Splitt, S. Lazarus, F. Martinez-McKinney, B. Hazelton, B. Grefenstette, A. Lowell, and H. K. Rassoul, Relativistic electron avalanches as a thun- derstorm discharge competing with lightning, Nat. Commun. 6, 7845 (2015).

[9] P. Kochkin, A. P. J. van Deursen, M. Marisaldi, A. Ursi, A. I. de Boer, M. Bardet, C. Allasia, J.-F. Boissin, F. Flourens, and N. Østgaard, In-flight observation of gamma ray glows by ILDAS, JGR Atmos. 122, 12801 (2017).

[10] V. Alexeenko, N. Khaerdinov, A. Lidvansky, and V. Petkov, Transient variations of secondary cosmic rays due to atmospheric electric field and evidence for pre-lightning particle acceleration, Phys. Lett. A 301, 299 (2002).

[11] A. Chilingarian, A. Daryan, K. Arakelyan, A. Hovhannisyan, B. Mailyan, L. Melkumyan, G. Hovsepyan, S. Chilingaryan, A. Reymers, and L. Vanyan, Ground-based observations of thunderstorm-correlated fluxes of high-energy electrons, gamma rays, and neutrons, Phys. Rev. D 82, 043009 (2010).

[12] A. Chilingarian, G. Hovsepyan, and A. Hovhannisyan, Particle bursts from thunderclouds: Natural particle accelerators above our heads, Phys. Rev. D 83, 062001 (2011).

[13] T. Torii, T. Sugita, M. Kamogawa, Y. Watanabe, and K. Kusunogi, Migrating source of energetic radiation generated by thunderstorm activity, Geophys. Res. Lett. 38, L24801 (2011).

[14] H. Tsuchiya, T. Enoto, K. Iwata, S. Yamada, T. Yuasa, T. Kitaguchi, M. Kawaharada, K. Nakazawa, M. Kokubun, H. Kato, M. Okano, T. Tamagawa, and K. Makishima, Hardening and Termination of Long-Duration Gamma Rays Detected Prior to Lightning, Phys. Rev. Lett. 111, 015001 (2013).

[15] Y. Kuroda, S. Oguri, Y. Kato, R. Nakata, C. Ito, and M. Minova, Observation of gamma ray bursts at ground level under the thunderclouds, Phys. Lett. B 758, 286 (2016).

[16] K. Kudela, J. Chum, M. Kollárik, R. Langer, I. Strhárský, and J. Baše, Correlations between secondary cosmic ray rates and 
strong electric fields at Lomnický štít, J. Geophys. Res. Atmos. 122, 10700 (2017).

[17] A. Gurevich, V. P. Antonova, A. P. Chubenko, A. N. Karashtin, G. G. Mitko, M. O. Ptitsyn, V. A. Ryabov, A. L. Shepetov, Yu. V. Shlyugaev, L. I. Vildanova, and K. P. Zybin, Strong Flux of Low-Energy Neutrons Produced by Thunderstorms, Phys. Rev. Lett. 108, 125001 (2012).

[18] H. Tsuchiya, K. Hibino, K. Kawata, N. Hotta, N. Tateyama, M. Ohnishi, M. Takita, D. Chen, J. Huang, M. Miyasaka, I. Kondo, E. Takahashi, S. Shimoda, Y. Yamada, H. Lu, J. L. Zhang, X. X. Yu, Y. H. Tan, S. M. Nie, K. Munakata, T. Enoto, and K. Makishima, Observation of thundercloud-related gamma rays and neutrons in Tibet, Phys. Rev. D 85, 092006 (2012).

[19] A. Chilingarian, N. Bostanjyan, and L. Vanyan, Neutron bursts associated with thunderstorms, Phys. Rev. D 85, 085017 (2012).

[20] T. Enoto, Y. Wada, Y. Furuta, K. Nakazawa, T. Yuasa, K. Okuda, K. Makishima, M. Sato, Y. Sato, T. Nakano, D. Umemoto, and $\mathrm{H}$. Tsuchiya, Photonuclear reactions triggered by lightning discharge, Nature (London) 551, 481 (2017).

[21] A. Gurevich, G. Milikh, and R. Roussel-Dupre, Runaway electron mechanism of air breakdown and preconditioning during a thunderstorm, Phys. Lett. A 165, 463 (1994).

[22] L. Babich, E. Donskoy, I. M. Kutsyk, A. Yu. Kudryavtsev, R. A. Roussel-Dupré, B. N. Shamraev, and E. M. D. Symbalisty, Comparison of relativistic runaway electron avalanche rates obtained from Monte Carlo simulations and from kinetic equation solution, IEEE Trans. Plasma Sci. 29, 430 (2001).

[23] L. Babich, E. Donskoy, R. Il'kaev, M. Kutsyk, and R. A. Roussel-Dupré, Fundamental parameters of a relativistic runaway electron avalanche in air, Plasma Phys. Rep. 30, 616 (2004).

[24] J. Dwyer, A fundamental limit on electric fields in air, Geophys. Res. Lett. 30, 2055 (2003).

[25] A. Chilingarian, B. Mailyan, and L. Vanyan, Recovering of the energy spectra of electrons and gamma rays coming from the thunderclouds, Atmos. Res. 114-115, 1 (2012).

[26] A. Chilingarian, G. Hovsepyan, Y. Khanikyanc, A. Reymers, and S. Soghomonyan, Lightning origination and thunderstorm ground enhancements terminated by the lightning flash, Europhys. Lett. 110, 49001 (2015).

[27] A. Chilingarian, S. Chilingaryan, and G. Hovsepyan, Calibration of particle detectors for secondary cosmic rays using gamma-ray beams from thunderclouds, Astropart. Phys. 69, 37 (2015).

[28] A. Chilingarian, G. Hovsepyan, and L. Kozliner, Thunderstorm ground enhancements: Gamma ray differential energy spectra, Phys. Rev. D 88, 073001 (2013).

[29] A. Chilingarian, Long lasting low energy thunderstorm ground enhancements and possible Rn-222 daughter isotopes contamination, Phys. Rev. D 98, 022007 (2018).
[30] V. Bogomolov, A. Iyudin, A. Maximov, M. I. Panasyuk, and S. I. Svertilov, Comment on "Long lasting low energy thunderstorm ground enhancements and possible Rn-222 daughter isotopes contamination", Phys. Rev. D 99, 108101 (2019).

[31] A. Chilingarian, A. Avetisyan, G. Hovsepyan, T. Karapetyan, L. Kozliner, B. Sargsyan, and M. Zazyan, Origin of the lowenergy gamma ray flux of the long-lasting thunderstorm ground enhancements, Phys. Rev. D 99, 102002 (2019).

[32] A. Chilingarian, Reply to "Comment on 'Long lasting low energy thunderstorm ground enhancements and possible Rn-222 daughter isotopes contamination'", Phys. Rev. D 99, 108102 (2019).

[33] M. Wilkening, M. Kawano, and Carlton Lane, Radon-daughter ions and their relation to some electrical properties of the atmosphere, Tellus 18, 679 (1966).

[34] Y. Reuveni, Y. Yair, C. Price, and G. Steinitz, Ground level gamma-ray and electric field enhancements during disturbed weather: Combined signatures from convective clouds, lightning and rain, Atmos. Res. 196, 142 (2017).

[35] N. Østgaard, H. J. Christian, J. E. Grove, D. Sarria, A Mezentsev, P. Kochkin, N. Lehtinen, M. Quick, S. Al-Nussirat, E. Wulf, G. Genov, K. Ullaland, M. Marisaldi, S. Yang, and R. J. Blakeslee, Gamma ray glow observations at 20-km altitude, J. Geophys. Res.: Atm. 124, 7236 (2019).

[36] D. Shi, D. Wang, T. Wu, and N. Takagi, Correlation between the first return stroke of negative CG lightning and its preceding discharge processes, J. Geophys. Res.: Atm. 124, 8501 (2019).

[37] A. Chilingarian, S. Chilingaryan, T. Karapetyan, L. Kozliner, Y. Khanikyants, G. Hovsepyan, D. Pokhsraryan, and S. Soghomonyan, On the initiation of lightning in thunderclouds, Sci. Rep. 7, 1371 (2017).

[38] W. Xu, S. Celestin, and V. P. Pasko, Optical emissions associated with terrestrial gamma ray flashes, J. Geophys. Res.: Space Phys. 120, 1355 (2015).

[39] http://www.moonglowtech.com/products/AllSkyCam/

[40] S. Chilingaryan, A. Chilingarian, V. Danielyan, and W. Eppler, The Aragats data acquisition system for highly distributed particle detecting networks, J. Phys. Conf. Ser. 119, 082001 (2008).

[41] A. Chilingarian, S. Chilingaryan, and A. Reymers, Atmospheric discharges and particle fluxes, J. Geophys. Res. Space Physics 120, 5845 (2015).

[42] https://cloud.mail.ru/public/3ZxZ/3N3bYnHPf/5555555.mp4

[43] J. Abraham et al. (The Pierre Auger Collaboration), The fluorescence detector of the Pierre Auger observatory, NIM 620, 227 (2010).

[44] http://www.nasa.gov/cassini and http://saturn.jpl.nasa.gov

[45] A. Chilingarian and H. Mkrtchyan, Role of the Lower Positive Charge Region (LPCR) in initiation of the Thunderstorm Ground Enhancements (TGEs), Phys. Rev. D 86, 072003 (2012). 